\title{
Further experiments on the perception of growth in three dimensions
}

\author{
VICKI BRUCE, MIKE BURTON, TONY DOYLE, and NEAL DENCH \\ University of Nottingham, Nottingham, England
}

\begin{abstract}
Mark and Todd (1983) reported an experiment in which the cardioidal strain transformation was extended to three dimensions and applied to a three-dimensional (3-D) representation of the head of a 15-year-old girl in a direction that made the transformed head appear younger to the vast majority of their subjects. The experiments reported here extend this research in order to examine whether subjects are indeed detecting cardioidal strain in three dimensions, rather than detecting changes in head slant or making 2-D comparisons of the shape of the occluding contour. Three-dimensional surfaces were obtained by measuring a real head manually (Experiment 1) and with a laser scanner (Experiment 2), and transformed to different age levels using the 3-D strain transformation described by Mark and Todd (1983). There were no statistically significant differences in the accuracy with which relative age judgments could be made in response to pairs of profiles, pairs of $3 / 4$ views, or pairs of mixed views (profile plus $3 / 4$ view), suggesting that subjects can indeed extract the cardioidal strain level of the head in three dimensions. However, an additional effect that emerged in these studies was that judgments were crucially affected by the instructions given to subjects, which suggests that factors other than cardioidal strain are important in making judgments about rich data structures.
\end{abstract}

Research conducted over the past 15 years by Pittenger, Shaw, Mark, and Todd (e.g., Mark \& Todd, 1985; Mark, Todd, \& Shaw, 1981; Pittenger \& Shaw, 1975a, 1975b; Pittenger, Shaw, \& Mark, 1979) has clearly established that human observers are sensitive to the cardioidal strain level of a human craniofacial profile when making judgments of its age. Cardioidal strain is a geometric transformation that models the actual changes that occur as an infant's face and cranium grow from infancy to adulthood. In polar coordinates $(R, \theta)$ the cardioidal strain transformation is given by the equation $R^{\prime}=R(1-k \cos \theta)$, $\theta^{\prime}=\theta$. Numerous experiments have shown that craniofacial profiles are rendered apparently older in appearance by applying the strain transformation in one direction, and younger by a transformation in the opposite direction (see Figure 1), as set by varying the size and sign of the free parameter $k$. These studies have also demonstrated that other geometric transformations that have equivalent effects on face angle do not produce the same effects in terms of perceived aging of the face. One such comparison transformation used in many of the studies has been affine shear (see Figure 1). In rectangular coordinates $(x, y)$, affine shear is given by the equations $y^{\prime}=\mathrm{y}, x^{\prime}=\mathrm{x}+y \tan \theta$, where the free parameter is the angle $\theta$.

A clear example of research in this field is provided by the experiments of Mark and Todd (1985). Since their

This research was supported by a grant from the Science and Engineering Research Council (GR/F 10569). We thank Alf Linney and Anne Coombes at University College, London, for providing their laser scanning facilities. Correspondence may be addressed to Vicki Bruce, Department of Psychology, University of Nottingham, Nottingham NG7 2RD, England. design has been partially replicated in the studies to be reported here, we will describe the design and results of their study in some detail. Mark and Todd (1985) used traced sets of profiles (cf. Mark et al., 1981) of different individuals, which were taken from longitudinal growth records spanning ages of about 6 to 20 years (see Figure 1, top row). The change in facial angle (see below) between the youngest and oldest actual profile was used to define the extent of cardioidal strain and other transformations applied to the youngest profiles from each patient to produce sets of artificial series that map onto those of actual growth. From the youngest profile of each individual, a sequence of four increasingly "older" profiles (defined by facial angle) was created, using a number of different transformations. The transformations (see Figure 1) included cardioidal strain and affine shear, which will form the focus of the present paper. Within each series of five profiles, 20 different pairs of different profiles were produced by pairing each profile with each other one. The pairs differed in age level by different numbers of steps, from one (adjacent profiles along a row in Figure 1) to a maximum of four (where the "youngest" profile was paired with the "oldest" in a row in Figure 1).

Mark and Todd (1985) asked subjects to decide which profile in each of a series of pairs looked the older, and they plotted out the rates of choosing the objectively "older" member of each pair (as defined by change in face angle in Figure 1) as a function of the number-ofsteps difference between the pairs, and as a function of the kind of transformation relating members of the pair. They found that actual growth, cardioidal strain, and spiral strain gave virtually identical psychophysical functions, with accuracy increasing from about $75 \%$ at the one-step 
ACTUAL GROWTH
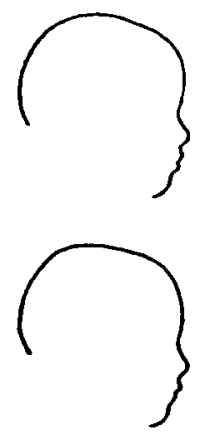

CARDIOIDAL STRAIN

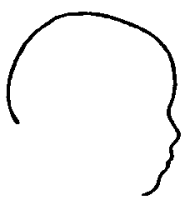

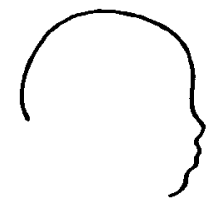
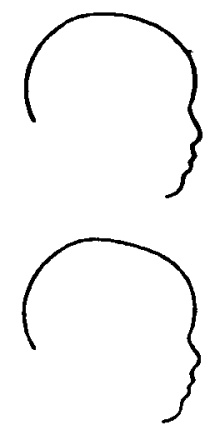
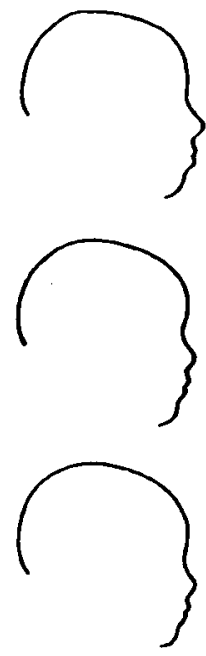
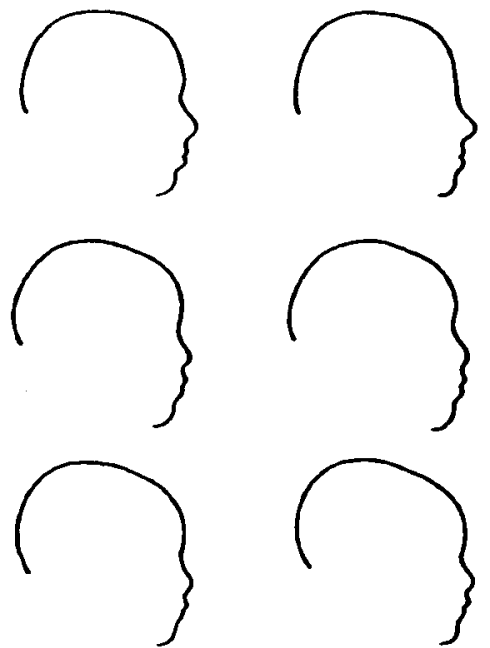

1

Figure 1. Examples of profile sequences resulting from actual growth, cardioidal strain, and afîne shear. From Mark, Todd, and Shaw (1981), used with the first author's permission. Copyright 1981 by the American Psychological Association, Inc.

difference to near ceiling at four steps. Performance on affine shear, reflected shear, and rotation was substantially lower, rising from around $60 \%$ at the one-step difference to $70 \%$ or more with four steps. Thus, the subjects seemed to respond to cardioidal strain in the same way, and with as great sensitivity, as they did to actual growth. Nevertheless, the subjects were also able to make judgments significantly above chance in response to profiles with shear or rotation applied to them. This appeared to be due to the actual change in face slant that was available for subjects to use to judge these images (as seen in Figure 1). In a second experiment, profiles were rotated back to the same face orientation for all age levels. Results remained very similar for growth and strain transformations (i.e., rising from about $75 \%$ to near $100 \%$ with increasing step size), but they collapsed toward chance for the shear and rotation transformations. In a final experiment, Mark and Todd (1985) demonstrated that in a simple same-different pattern-matching task, the subjects were as accurate in detecting a difference between, say, two profiles related by a shear transformation as between two related by a strain transformation, showing that the differences obtained in the relative age experiments were due not merely to differences in the perceptibility of different changes, but specifically to the association of these changes with the effects of growth.

It has been shown in other work that human observers appear to be sensitive to cardioidal strain as an abstract deformation that is largely independent of the nature of the structure undergoing the change. Thus, for example, Pittenger et al. (1979) showed that strain could be applied to pictures of Volkswagen cars, birds, and dogs to render these apparently older and younger to (but see Mark, Shapiro, \& Shaw, 1986, as well as below in this paper, for important qualifications). Cardioidal strain thus seems to be a good candidate for an "invariant" to which hu- mans are directly sensitive when making judgments of relative and absolute age.

Although such studies have involved pictures of human profiles, cartoon cars, and pictures of birds and dogs, it is nevertheless the case that the majority of studies of the perception of cardioidal strain level have made use of highly schematic and impoverished materials like those shown in Figure 1, rather than more realistic depictions of human faces. In 1983, Mark and Todd reported an interesting extension of this work to examine the effects of a three-dimensional (3-D) strain transformation to a representation of the surface of a face and head. In their study, a 3-D database of the face and head of a 15-yearold girl was constructed by the Solid Photography Studio, and the database was used to drive a lathe that carved a bust of the girl. The database was also transformed, using the cardioidal strain equation extended to 3-D (in spherical coordinates $R, \theta, \phi ; R^{\prime}=R[1-k \cos \theta], \theta^{\prime}=\theta$, $\phi^{\prime}=\phi$ ), with $k$-the free parameter that determines the growth level of the resulting head-set to -0.2 , which should result in an an apparently younger head. A further computer sculpture was produced from the transformed database, and the two busts were shown to observers "from a variety of perspectives." Of 360 observations, 356 put the original bust as "older," and the actual age assigned to that bust was 14.5 years, compared with the girl's actual age of 15 years. The actual age assigned to the "younger" bust was given as 6.3 years, and people who knew the child said that the bust closely resembled her at about 5 years old, although it lacked some of the "puppy fat" associated with that age.

Mark and Todd's ambitious demonstration clearly suggests that cardioidal strain level may be seen in three dimensions. However, the study on its own is inconclusive, since it is not clear on what basis the subjects made their judgments. In this respect, there were two major 
deficiencies in the study. First, the transformed head was not corrected for head slant, so that observers may have based their judgments on this rather than on the more abstract "cardioidal strain" level. Second, from the description in the paper, it seems that the views to be judged were not controlled-so we do not know whether or not subjects could make their judgments by comparing the occluding contours of coincident viewpoints. The aim of the experiments reported here was to reexamine the perception of relative age in three dimensions, using carefully controlled viewpoints and head angles. To do this, we made use of a graphics workstation on which to manipulate and display 3-D surface representations of human heads.

\section{GENERAL METHOD}

A single 3-D database of an adult female head (V.B.) with hair concealed by a swimming cap was obtained by measurement from photographs (Experiment 1 ) and by laser scanning (Experiment 2). Head models were displayed and rendered on a SUN 3/110 gray-scale workstation, using Suncore graphics tools. The set of $x-, y-$, $z$-coordinate points obtained from each head was faceted to form a 3-D polygonal surface to which a standard lighting model could be applied to produce a smooth surface that looked like a milliner's dummy (Gouraud shading was used in all the work to be reported here). The resulting head model could be displayed in any orientation, but in these experiments, orientations were confined to full profile and 3/4 view (see Figures 2 and 3). In order to transform this surface in various ways, we placed the origin of the coordinate system midway between the earholes, with the horizontal axis lying parallel to a line joining the top of the earholes to the bottom of the eye socket. This is known as the Frankfurt horizontal. Face angle was measured as the angle between the Frankfurt horizontal and a line joining the bottom of the deepest depression in the profile just above the nose under the eyebrow and the highest point of the chin. Face angle was measured and used to equate the degree to which the strain and shear transformations were applied, but all faces were rotated back to render this line vertical in the stimuli whose ages were to be judged.

The same experimental design was used in all three studies, and it closely followed that reported by Mark and Todd (1985). In each study, a between-groups design was used, in which different subjects judged the relative ages of different views and transformations. The betweengroups design was used despite its lesser sensitivity, for we did not want judgments of the age of one view of a face to be contaminated by those made in response to different views. Three of these groups of subjects judged pairs of faces whose age levels were manipulated using the strain transformation. The first group viewed pairs of profile faces, the second group viewed pairs of 3/4-view faces, and the third group viewed pairs showing one full face and one 3/4-view face. A fourth group of subjects viewed profile faces whose differences were produced by applying an affine shear, rather than cardioidal strain, in the manner described below.

To make the images for the experiment, from the starting, adult face (which we will call Face A), four further cardioidal-strained versions were produced in the younger direction by setting $k$ to $-0.07,-0.14,-0.21$, and -0.28 (which we will call Faces $B, C, D$, and $E$, respectively). (These values were chosen after informal experimentation to choose a range that encompassed the $k=-0.2$ value used by Mark and Todd [1983], while avoiding floor effects at the smallest step sizes. However, our choice of these four values extends the range of the strain transformation beyond the level used in much other research in this area. Possible implications of this difference will be discussed toward the end of this paper.) Four levels of affine shear were created, to mimic analogous levels of cardioidal strain, by shearing the head to give the same effect on face angle as did each of the above strain levels.

The subject's task was to decide which of two faces at two different "age" levels looked the younger. Like Mark and Todd (1985), we attempted to obtain psychophysical functions for these judgments, so that we could see whether subjects judging age differences between pairs of heads shown at different angles (3/4 view and profile) were any less sensitive to the transformation than those judging pairs of the same head angle (both $3 / 4$ view or both profile). These psychophysical functions were established by showing subjects a range of age differences that should have been relatively easy or difficult to judge in terms of the extent of the transformation difference shown between the two heads. In each of the four experimental conditions in which faces at levels A, B, C, $D$, and $E$ were available, the subjects saw 4 pairs of heads that were four steps apart (i.e., $4 \mathrm{~A}-\mathrm{E}$ pairs), 8 pairs that were three steps apart (4 A-D pairs and 4 B-E pairs), 12 pairs that were two steps apart (4 of each of the pairs A-C, B-D, and C-E), and 16 pairs that were only one step apart (four of each of the pairs A-B, B-C, C-D, and $D-E)$. Within each condition, the positions of the younger and older heads were balanced, and in the mixed condition (3/4 view with profiles), whether the younger or older head was shown in $3 / 4$ view was also balanced in the series of images. The total of 40 test pairs was shown to the subjects in a random series, with the subjects asked to indicate the "younger" face of each pair.

\section{EXPERIMENT 1}

This was a preliminary experiment in which we made use of a rather crude 3-D model of a human head. We report it here because its results are very consistent with those of the main study, and because the pattern of findings had some influence on the main study's design.

\section{Method}

Surface points were recovered from a set of photographs of the real head divided into triangles marked with an eyebrow pencil (cf. Parke, 1975; Waters, 1987). The hair was covered with a latex 
swimming cap and was allowed to lie flat obscuring the rear of the neck, which prevented too much distortion to the shape of the rear part of the head. Photographs were taken at $45^{\circ}$ intervals around this head. The points where the triangles meet were measured on the photos, and after the differences between the views had been reconciled, the points were entered into a single database suitable for the shading program. Errors crept into this measurement of the face surface from a number of different sources, which included the thickness of the lines, perspective distortions, and difficulty in locating the earholes in the photographs. In addition, the sparsity of triangles actually measured means that the underlying database gives only a very crude approximation to a smooth surface-the resulting head when rendered looks distinctly alien (see Figure 2).

Transformations to this surface database and pairs of images were created as was described in the General Method section. The images were Gouraud shaded and then printed, using a laserwriter, which resulted in a further loss of image resolution. Each subject was shown $\mathbf{4 0}$ pairs and asked to write down which member of each pair looked "the younger or more babyish in shape." It was explained that all heads were approximately the same size, and that it was differences in shape that they should judge. Twelve undergraduate subjects served in each of the four experimental conditions, judging pairs of profiles, $3 / 4$ views, and mixed views in the strain condition, and profiles in the affine shear condition.

\section{Results and Discussion}

The average "correct" judgments of younger heads are shown as a function of step difference, view, and transformation in Table 1. This table shows that, as expected, subjects in the strain conditions were generally more accurate with greater step size. A 3 (views) $\times 4$ (steps) mixeddesign analysis of variance (ANOVA) conducted on the data from the three strain conditions alone showed a significant main effect of steps $[F(3,99)=3.741, p<.05]$ and no other significant effects. The apparent advantage of $3 / 4$ views in this experiment is clearly not significant, and, more importantly, there is no evidence for a drop in performance in the mixed condition. Performance on the shear condition shows a different pattern to that in strain, with subjects' responses, if anything, showing a tendency to respond "younger' to the faces whose face angles map onto "older" members of the strain series. A 4 (conditions) $\times 4$ (steps) mixed-design ANOVA conducted on the data from all four conditions including shear showed a significant effect of conditions $[F(3,44)=4.154$, $p<.05$ ], qualified by a highly significant interaction between condition and steps $[F(9,132)=3.728, p<.001]$, which confirmed this impression.

This experiment thus generally confirmed that subjects respond differentially to the cardioidal strain transformation when asked to make judgments of relative age, and it seems that they are as able to make comparative decisions with respect to two heads in different views as they are with respect to two heads shown in a single view. However, performance overall in the strain condition never approached the ceiling found by Mark and Todd (1985) with drawn profiles, even though the extent of the transformations used in our study surpassed that of Mark and Todd in terms of its effect on face angle. These lower average performance levels conceal inconsistency in the way that individual subjects chose to assign heads to the category "old." Examination of the individual data in each condition revealed that a minority of subjects assigned their age judgments in a direction opposite to that predicted. In each of the strain conditions, about half the subjects scored $100 \%$ on one or both of Step Sizes 3 and 4 , but in each condition, there were also some subjects who scored $0 \%$ on the greatest step size and around $20 \%-25 \%$ on the smallest step size, apparently labeling the dimension in the "wrong" direction. One of these same subjects, on debriefing, claimed that they had thought the "youngest" heads looked like "really old bald men with scraggy chins." This suggested the possibility that the richer database used in this study might present a range of alternative features to subjects, which might lead them to assign age on the basis of these differing features. One problem with the images was that the coarse-
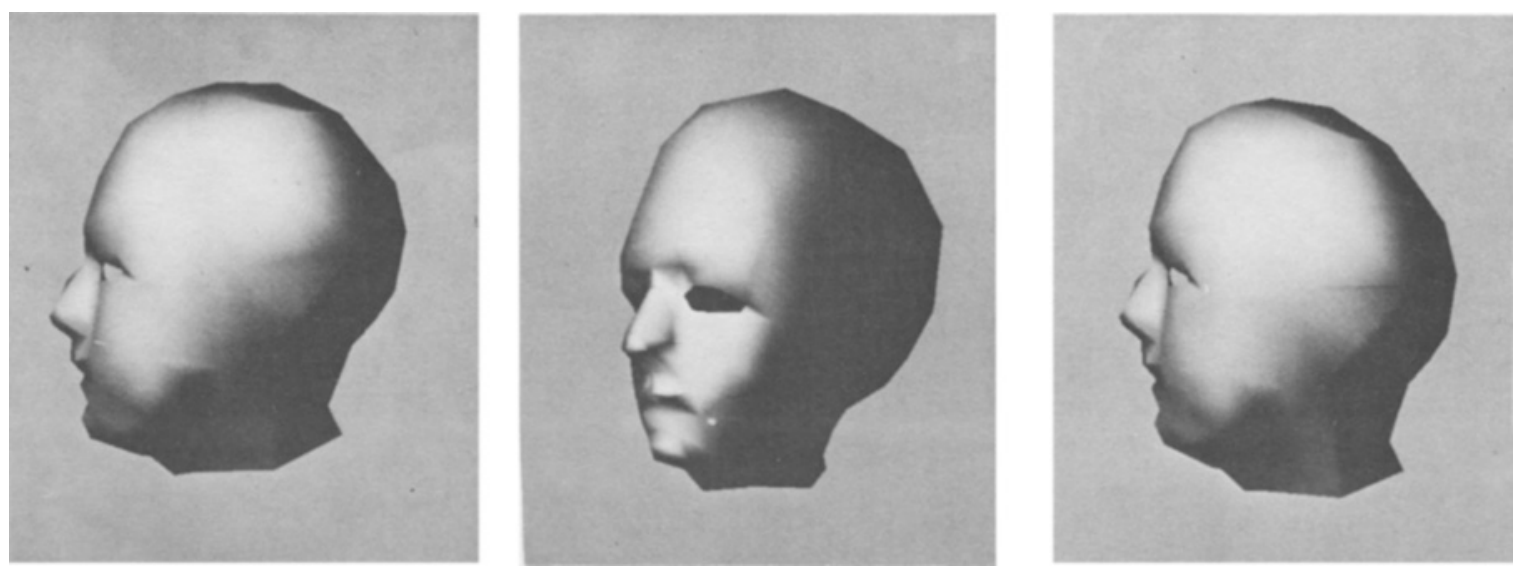

Figure 2. Examples of the heads used in Experiment 1. The left panel shows a profile view of the untransformed head. The central panel shows a $3 / 4$ view of the head strain-transformed by three steps $(k=-0.21)$. To the right is shown the third step of afine shear applied to the profile. 
Table 1

Mean Percent Correct Responses in Each Condition of Experiment 1

\begin{tabular}{lccccc}
\hline & \multicolumn{5}{c}{ Steps } \\
\cline { 2 - 5 } \multicolumn{1}{c}{ Conditions } & 1 & 2 & 3 & 4 & Mean \\
\hline Strain profiles & 58 & 72 & 64 & 67 & 65 \\
Strain 3/4 views & 75 & 71 & 81 & 79 & 77 \\
Strain mixed & 60 & 73 & 78 & 75 & 72 \\
Shear profiles & 53 & 42 & 36 & 33 & 41 \\
\hline
\end{tabular}

ness of the 3-D sampling points combined with the rendering made some of the "younger" heads look rather alien in form. To examine this, a further 12 subjects were tested in a condition in which the profiles used in the experiment were shown in silhouette, concealing all "shape" information except that contained in the occluding contour. Performance remained very similar to that seen in the original experiment-averaging $65 \%, 73 \%$, $77 \%$, and $79 \%$ at Steps 1, 2, 3, and 4, respectively. Again, a minority of the subjects appeared to make the age assignment in the "reverse" direction.

At this point, we felt it was sensible to seek a better, smoother, head model and replicate the experiment, to see whether or not the inconsistency in our subjects was a product of the roughness of the model. Mark et al. (1986), for example, found that cardioidal strain transformations were only readily seen as reflecting growth or aging when applied to a smooth head, and our head had a certain angularity due to the sparse database underlying it. However, we felt that the subjects' inconsistency might also reflect something more important about the use of an adult head in transformations more appropriate to childhood. In addition, given the way in which some subjects chose to interpret the relative age range produced by transforming an adult head as referring to a much "older" range than that expected, we thought it worthwhile to explore the effect of explicit manipulation of subjects' instructions in the task. The next experiment was run in two parts, with half the subjects explicitly told that some of the heads should appear more "babyish" in shape, and shown examples of adult and children's heads before the experiment. The remaining subjects were given no such direction, and merely asked to indicate which head looked "younger."

\section{EXPERIMENT 2}

\section{Method}

Materials. In this experiment, we used an $x-, y$-, $z$-coordinate description of the external surface of the head of the subject used in the previous experiment. This description was obtained from a laser scan, using equipment that has been developed to create models from which plastic surgeons can assess the possible effects of plastic surgery (cf. Moss, Grindrod, Linney, Arridge, \& James, in press a; Moss, Linney, Grindrod, \& Mosse, in press b). During the scan, the subject (V.B.) wore a tight-fitting swimming cap over the hair. The original head description had 27,000 coordinate points, but this was reduced to approximately a quarter of these (by systematic elimination of alternate points around each profile and crosssection) to reduce the storage demand during stimulus preparation.
In normal use, the front part of the face only is recorded, and the subject's head is tilted well back to ensure that the front part of the chin does not cast a shadow on the upper neck and jaw. This created some difficulties for the $360^{\circ}$ scan needed in the present study; the resulting model had a patch missing from one side of the head above one ear and the very top of the head was absent. The missing part was patched by reconstructing the top of the data slices involved so that they matched the complete slices on either side. The top of the head was rebuilt so that the slices were all carried up to the center of rotation, obeying rules to ensure that each slice passed through the top of the real head as found by manual measurement. Standard spline functions were used to patch the top smoothly to produce a new top, which was correct in its maximum dimensions and blended in well with the orginal head. Several slight vertical ridges caused by the wobbling of the revolving chair during the laser scan were carried up by this patching technique from the forehead to the crown. These were noticeable but were no bigger than wrinkling at the rear caused by the swimming cap. (Sophisticated smoothing of these wrinkles would have been very timeconsuming, while other smoothing methods would have reduced the detail of other parts of the face. Possible implications of the visible wrinkles are discussed later in the paper.) The resulting database was displayed and transformed as before, except that photographs were taken from the screen of a Gouraud-shaded model, which were then duplicated to form pairs of images for the experiment (see Figure 3 for examples).

Procedure. The subjects were shown pairs of images. It was explained to them that these images were computer-generated from a model wearing a swimming cap (to explain the wrinkles now evident in this high-resolution surface model). Half the subjects were asked to state which member of each pair was the "younger" in shape, but no reference was made to the "babyish" shape we expected them to see. It was again stressed that it was differences in shape, not size, that they were expected to see. The other set of subjects were told to mark down which head looked younger or more babyish in shape in comparison with the other, which they were told ought to look older or more adult in shape. To direct them to consider the difference between adult and baby-shaped heads, three assorted magazine photographs were appended showing celebrities with offspring about 2-7 years old.

A total of 120 undergraduate and local college subjects were tested in the experiment, with 15 tested in each of the three strain conditions and one shear condition, for each kind of instruction.

\section{Results and Discussion}

The average percent correct responses in each condition of the experiment are shown in Table 2, which shows the results obtained from unconstrained and constrained ("babyish") instructions. It is evident that performance in this experiment with unconstrained instructions is now even further away from the accurate judgments expected at the larger step differences. Performance with specific mention of "babyish" head shape and pictured examples is now much more as expected, though it still does not reach ceiling at the larger step sizes. An initial 2 (instructions) $\times 4$ (conditions: strain profile, $3 / 4$, mixed, and shear) $x 4$ (steps) mixed-design ANOVA revealed a main effect of instructions $[F(1,112)=6.681, p<.05]$ and of conditions $[F(3,112)=10.319, p<.001]$, as well as an interaction between conditions and instructions $[F(3,112)=6.377, p<.001]$. The interaction between number of steps and instructions was also significant $[F(3,336)=2.646, p<.05]$. Clearly the pattern of ef- 

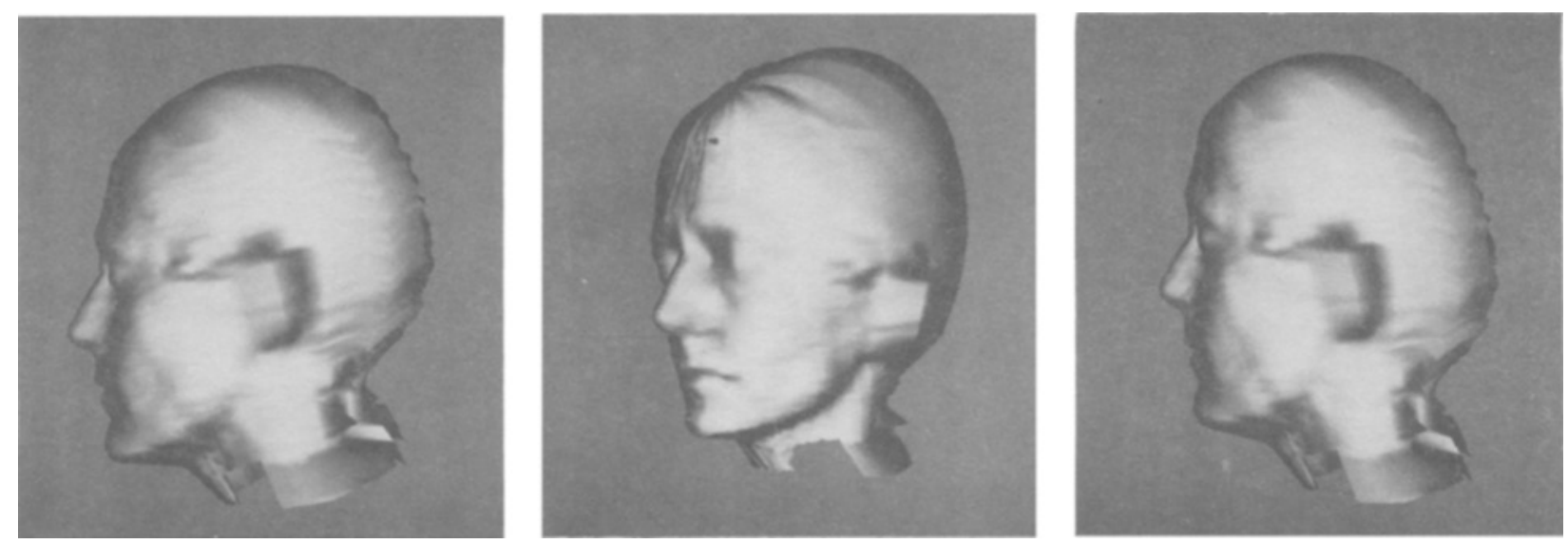

Figure 3. Examples of the heads used in Experiment 2. The left panel shows a profile view of the original head, and the central panel shows a $3 / 4$ view of the head strain-transformed by three steps $(k=-0.21)$. To the right is a profile version, which shows the third step of affine shear.

fects varies according to the instructions given subjects. In order to pursue these effects further, separate analyses were conducted on the data arising from each set of instructions in the experiment.

Unconstrained instructions. A 4 (conditions) $\times 4$ (steps) mixed-design ANOVA showed no significant effects of conditions or steps (all $p s>.2$ ). The variance within each condition was quite large, and, as in Experiment 1 , it arose because the subjects assigned their judgments to different ends of the dimensions. The subjects in the three strain conditions appeared to do this to a greater extent than those in the shear condition. For example, comparing the strain profile with the shear profile conditions (which both apparently average "chance" performance from the means for unconstrained instructions in Table 2), 14 of the 15 subjects scored either $0 \%$ or $100 \%$ on the 4-step pairs in the strain condition (expected frequency on chance responding is $12.5 \%$ ), whereas only 7 of the 15 subjects in the shear condition scored $0 \%$ or $100 \%$ in the 4 -step condition.

Constrained ("babyish") instructions. A 4 (conditions) $\times 4$ (steps) mixed-design ANOVA showed significant ef-

Table 2

Mean Percent Correct Responses in Each Condition of Experiment 2

\begin{tabular}{lccccc}
\hline & \multicolumn{5}{c}{ Steps } \\
\cline { 2 - 5 } \multicolumn{1}{c}{ Conditions } & 1 & 2 & 3 & 4 & Mean \\
\hline \multicolumn{5}{c}{ Unconstrained } & Instructions \\
Strain profiles & 48 & 45 & 43 & 45 & 45 \\
Strain 3/4 views & 70 & 72 & 64 & 60 & 66 \\
Strain mixed & 50 & 61 & 59 & 55 & 56 \\
Shear profiles & 48 & 54 & 50 & 52 & 51 \\
& Constrained Instructions & & \\
Strain profiles & 68 & 74 & 81 & 82 & 76 \\
Strain 3/4 views & 77 & 82 & 87 & 88 & 84 \\
Strain mixed & 65 & 77 & 83 & 85 & 78 \\
Shear profiles & 37 & 30 & 24 & 25 & 29 \\
\hline
\end{tabular}

fects of conditions $[F(3,56)=26.8, p<.001]$, steps $[F(3,168)=4.31, p<.01]$, and their interaction $[F(9,168)=3.68, p<.001]$. This interaction arose because subjects in the shear condition assigned their age judgments in a direction opposite to that predicted on the basis of face angle alone-they showed a tendency to see heads at the older end of the transformation as being younger. If the data from the three strain conditions are analyzed together, only a significant effect of steps is seen $[F(3,126)=11.134, p<.001]$, and there is no significant effect of conditions within strain $(F<1)$.

It seems that the inconsistency shown by the subjects in Experiment 1 cannot be attributed simply to the poverty of the head model used there; in Experiment 2 a better head model was used, but the results depart even further from those expected. It may be that, when an adult head model is used as the starting point for a series of transformations, it is important to specify the range of age changes one expects subjects to see. The unconstrained instructions may lead some subjects to look for changes between a 30-year-old and an 80-year-old head, rather than the changes associated with growth over a much younger age range. Constraining the instructions and directing the subjects to seek evidence of "babyish" shapes reduces, but does not eliminate, this tendency. Performance asymptotes at about $85 \%$ accuracy in the 3- and 4-step conditions because some subjects in each of the strain conditions failed to see the aging transformation in the expected direction.

A further, unexpected, difference between our results and those reported elsewhere (e.g., Mark \& Todd, 1985) is that our subjects did assign an age "direction" to the sheared profile heads with some consistency. The direction is opposite to that which would arise from some detection of change in face angle, and it may arise because the greater the shear applied to the head, the flatter the back of the head appears once the face slant has been corrected 
back to vertical (see Figure 3). Thus, less sheared faces have more rounded (babyish) backs. Under unconstrained instructions, the subjects were completely inconsistent in their judgments of the shear condition (Table 2), but the mention of "babyish" shapes (Table 1 and especially Table 2) seems to have drawn their attention to this feature.

These observations notwithstanding, the results of Experiment 2 do confirm those of Experiment 1 and suggest that subjects can make relative age judgments as readily across different views as within them. It is also worth noting that the mean results for both instructional conditions again show a nonsignificant advantage for the 3/4-view condition over the other two strain conditions-a result that parallels the nonsignificant trend found in Experiment 1 with the coarse head.

\section{GENERAL DISCUSSION}

The experiments reported here generally support the work of Mark and Todd (1983) in showing that cardioidal strain level can be applied, and perceived, in the three dimensions of more realistic facial surfaces. In two experiments, subjects were as good at making relative age judgments in response to two faces shown in different head angles (profile and 3/4 view) as they were in response to two heads shown in the same angle, in which case the shapes of occluding contours could be compared directly. Additionally, there was a nonsignificant trend in both experiments for $3 / 4$ views to be compared somewhat more easily than profiles, which is consistent with other demonstrations of advantages in matching 3/4 views of unfamiliar faces (e.g., Bruce, Valentine, \& Baddeley, 1987). However, our findings of the inconsistency of some subjects in judging age in the manner expected, as well as the extreme sensitivity of these judgments to instructions, suggests some qualifications to the idea that cardioidal strain is an abstract invariant that may be applied to a range of structures with equivalent results. Our findings show that an adult head, age-transformed to a younger level, is not invariably seen as younger-only when instructions encourage subjects to focus on the level of age during which growth occurs do judgments coincide with those predicted.

A similar sensitivity to instructions was noted by Mark et al. (1981), who showed subjects series of pröfiles such as those in the three rows of Figure 1, and asked subjects to describe the style of change (e.g., gaining weight, growing) that best characterized the process that produced the change in the profiles from left to right. In both reported studies, subjects were much more likely to mention growth if the series shown was that obtained from actual growth or from strain transformations than if it resulted from a transformation such as affine shear, which is unrelated to aging. However, this tendency to mention growth or aging as the style of change characterizing actual growth and strain sequences was much increased when instructions specifically mentioned growth as a possible response category.
Mark et al. (1986) also demonstrated situations in which cardioidal strain had reduced impact on perceived age. In their studies, the angularity of schematic heads was varied, from a "robot" head composed of straight lines and angles, to a smoother "human" head. They found that the effects of strain were qualified by those of angularity-only the smoother heads were seen as "growing." Their study is particularly relevant here, since our first experiment used a rather robot-like (i.e., angular) head model, and thus their research provided a possible explanation for the inconsistency in age judgments regarding this head. However, although in Experiment 2 we used a much smoother head, when the instructions were unconstrained, the results departed even more from those expected, so the angularity of our "rough" head model cannot provide the sole explanation.

Mark et al.'s (1986) study is also relevant because the schematic head profiles varied were clearly those of adults rather than children. Given a smooth adult profile, their subjects perceived the strain transformation in the predicted way. In other ongoing research by our own group (Bruce, Doyle, Burton, \& Dench, 1989), we have shown that applying a pseudostrain transformation to the positions of facial features within clearly adult, schematic face images (using a Mac-a-Mug electronic face composite system) produces highly systematic, though small, effects of the perceived age of these adult faces. It thus cannot be merely that the domain of application of the strain transformation is limited to "children's" faces; we suspect, rather, that a number of additional factors may come into play once a richer database is available for inspection.

In this respect, research by Mark et al. (1980) showing the interaction of cardioidal strain level with facial wrinkles is important. They took a series of profiles drawn by the artist Burne Hogarth depicting a man at 15, 30, 50 , and 70 years of age. These profiles contained varying shapes and also varying wrinkles with age. They traced the profile shapes and reproduced each of the age shapes with each of the four possible levels of wrinkle, and asked the subjects to assign ages to each of the 16 resulting images. Mark et al. (1980) found that when wrinkles appropriate to a 50 - or 70-year-old were added to the face, the age-related changes in shape had little impact on perceived age level, which was dominated by the heavy wrinkling. However, when wrinkle level was appropriate to the 15 - or 30-year-old, then shape-related changes in age were seen. In our experiments, the head model was measured from an adult aged in her $30 \mathrm{~s}$, and transformed in a direction that should produce shapes associated with younger ages. As Mark et al. (1980) point out, growth-related changes are about complete at age 30 , and so it makes sense only to transform the head in a direction that covers the period when growth would have occurred. We were, then, working within a range over which facial growth occurs, and so the strain transformation was applied legitimately. Nevertheless, our cardioidal transformation had little effect on the shape of the nose, for example, which may have signaled an adult head throughout the range of transformations applied. 
It is also possible that subjects' spontaneous attributions of "baldness" to the model may have led them to see the model as considerably older than she is. This in turn may have led them to perceive smaller chins as arising from tooth loss, as occurs at much greater ages, and it could have biased a proportion of them to perceive the relative ages the "wrong" way around. The wrinkling evident on the head used in Experiment 2 might also have contributed to this tendency. To check this, as part of a different research project (Bruce et al., 1989), 20 naive subjects were shown full-face and 3/4 views of the head used in Experiment 2, along with similar views of a (somewhat smoother) head of a younger, male adult (see Figure 4). The mean age assigned to the female head was 33 years $(S D=7$; the actual age at time of laser scan was 35 years) and to the male head 25 years $(S D=6$; actual age, 29 years). There is thus no evidence that the absolute ages of the untransformed head models are wrongly perceived, nor that the wrinkling evident on the female head created greater variability in the ages assigned.

A further point about the current experiments is that we used a greater range of strain transformation (to $k=-0.28$ ) than that used in other research in this area (where a maximum of $k=-0.2$ has been usual). It is possible that the presence of some heads whose global shape may be distorted beyond anything that could be natural has led subjects to make their judgments on some more local basis. We do not think this likely, since when

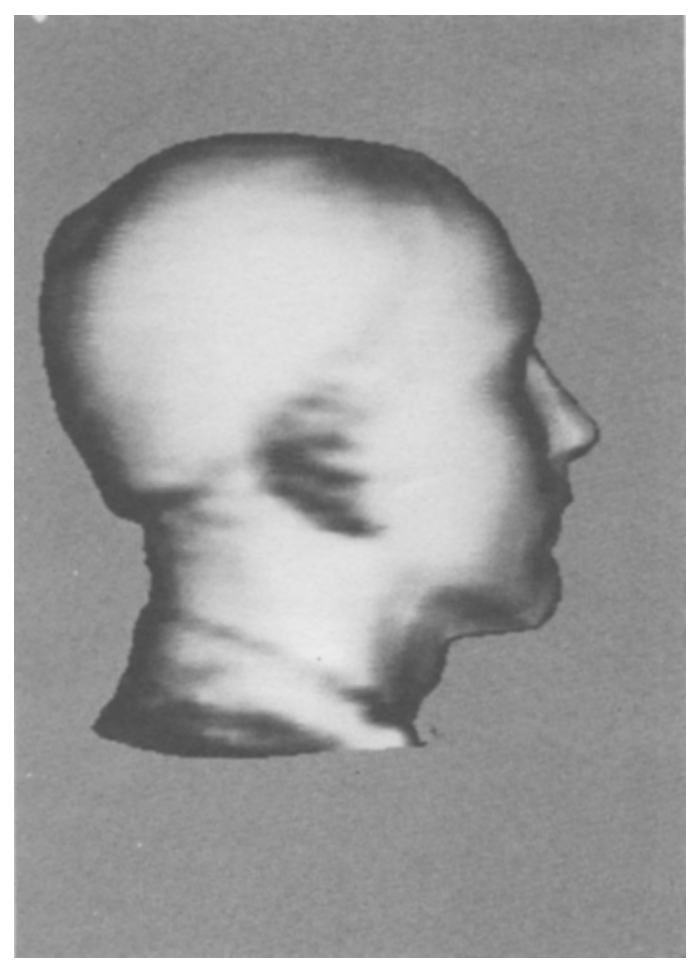

Figure 4. An example of a profile view of the smoother male head used in ongoing research. untransformed male and female heads (like those shown in Figures 3 and 4 but with systematically varied nose shapes) were embedded in a sequence that included straintransformed versions with $k$ set to -0.14 or -0.28 , very similar mean absolute ages were obtained with respect to the untransformed heads from 12 further subjects ( 34 years for the female head and 25 years for the male, averaging across the different nose shapes; Bruce et al., 1989). The results of this study additionally confirmed that naive subjects are very inconsistent in their assignments of age to heads transformed to different extents (even on the smoother, younger, male head). We do not feel, then, that the inconsistency of subjects' responses in the present paper, or the effect of instructions here observed, are simply an artifactual consequence of the quality of the stimuli or of the range of experimental variables tested.

This discussion, along with our observations of the effect of affine shear in our experiments, leads us to consider the underlying basis of relative age judgments within such an experiment. One possibility is to suggest, like Pittenger et al. (1979), that subjects are sensitive to the abstract transformational invariant-cardioidal straincharacterizing a particular style of change-growth. Given this perspective, these experiments suggest that this invariant can be perceived in three dimensions, but its perception may be influenced by other age-related changes (e.g., the size of the nose or baldness) that the subjects also know about. Another possibility is to suggest that growth (and, by analogy, cardioidal strain) is accompanied by changes to certain facial features and their topography. For example, children's features are relatively lower in the face than adults', so their foreheads appear disproportionately larger and their chins smaller. On this view, cardioidal strain leads to covariant changes in facial structures, but it is these, rather than the abstract "strain level," to which a viewer is sensitive. This perspective (which might be construed by some as merely a change in emphasis) may explain why the subjects in our experiments did show some consistency in judging shear when alerted to the relative shapes of adult and children's heads, and it may also explain why certain kinds of inconsistency arose in the effects of strain, depending on which features the subjects attended to. Such assessment of the relative sizes and placements of features might be easier from a $3 / 4$ view of the face, which reveals more of the face itself, and this could explain the (admittedly nonsignificant) trends for a 3/4-view advantage in our studies. This analysis would suggest that it is the effects of growth rather than growth itself that people perceive, which makes it easier to consider the nature of face perception in computational as well as ecological terms (cf. Bruce, 1988).

\section{REFERENCES}

BRUCE, V. (1988). Recognising faces. London: Erlbaum.

Bruce, V., Doyle, T., Burton, M., \& Dench, N. (1989). Effects of global and local facial transformations on judgments of age and sex. Manuscript in preparation. 
Bruce, V., Valentine, T., Baddeley, A. (1987). The basis of the $3 / 4$ view advantage in face recognition. Applied Cognitive Psychol. ogy, 1, 109-120.

Mark, L. S., Pittenger, J. B., Hines, H., Carello, C., Shaw, R. E., * ToDD, J. T. (1980). Wrinkling and head shape as coordinated sources of age-level information. Perception \& Psychophysics, 27, 117-124.

MARK, L. S., Shapiro, B. A., \& Shaw, R. E. (1986). Structural support for the perception of growth. Journal of Experimental Psychology: Human Perception \& Performance, 12, 149-159.

MARK, L. S. \& TonD, J. T. (1983). The perception of growth in three dimensions. Perception \& Psychophysics, 33, 193-196.

MARK, L. S., \& TODD, J. T. (1985). Describing perceptual information about human growth in terms of geometric invariants. Perception \& Psychophysics, 37, 249-256.

MARK, L. S., TODD, J. T., \& SHAw, R. E. (1981). Perception of growth: A geometric analysis of how different styles of change are distinguished. Journal of Experimental Psychology: Human Perception \& Performance, 7, 855-868.

Moss, J. P., Grindrod, S. R., Linney, A. D., Arridge, S. R., \& JAMES, D. (in press a). A computer system for the interactive planning and prediction of maxillo-facial surgery. American Journal of Orthodontics.
Moss, J. P., Linney, A. D., Grindrod, S. R., \& Mosse, C. (in press b). A laser scanning system for the measurement of facial surface morphology. Optics \& Lasers.

PARKE, F. I. (1975). Measuring three-dimensional surfaces with a twodimensional data tablet. Computers \& Graphics, 1, 5-7.

Pittenger, J. B., \& SHAw, R. E. (1975a). Aging faces as viscal elastic events: Implications for a theory of nonrigid shape perception. Journal of Experimental Psychology: Human Perception \& Performance, 1, 374-382.

Pittenger, J. B., \& Shaw, R. E. (1975b). The perception of relative and absolute age in facial photographs. Perception \& Psychophysics, $18,137-143$.

Pittenger, J. B., Shaw, R. E., MARK, L. S. (1979). Perceptual information for the age level of faces as a higher order invariant of growth. Journal of Experimental Psychology: Human Perception \& Performance, 5, 478-493.

WATERS, K. (1987). A muscle model for animating three-dimensional facial expression. Computer Graphics, 21, 17-24.

(Manuscript received February 6, 1989; revision accepted for publication May 15, 1989.) 